\title{
Santé publique, santé privée, rôles de l'État et du citoyen
}

\author{
Public and private health systems: the roles of the State and the individual
}

\author{
M.-F. Bacqué
}

(C) Springer-Verlag France 2011

\section{De l'expert à l'avocat}

La question du Médiator ${ }^{\circledR}$ en France a soulevé une vague de fond. Ont été remis en cause l'agence publique d'évaluation des médicaments et des produits de santé (Afssaps), les experts de ces produits, certains journaux scientifiques censés publier les études sur les médicaments. Le travail de la revue Prescrire $^{\circledR}$ a été à plusieurs reprises souligné : les valeureux rédacteurs avaient décrit depuis plusieurs années l'absence d'intérêt de ce médicament, pire, ses dangers présents et à venir. Les patients se sentent pris au piège, ligotés par leur incompétence en termes d'expertise biologique, mais surtout trahis par un système, le système de santé publique. Faudra-t-il que chaque citoyen devienne à son tour expert pour juger de l'innocuité d'un traitement ? Faudra-t-il un lourd appareil de chercheurs, de médecins, puis d'experts, de critiques et enfin d'avocats pour se soigner sans perdre sa santé ?

Le doute est instillé depuis longtemps aux États-Unis et nous renvoie à cette solitude devant la connaissance que l'on n'a pas et à la panique quand il s'agit de prendre une décision, pire, à l'amertume lorsque l'on s'est engagé dans une voie risquée et que l'on a perdu.

\section{La santé, un bien public qui ne peut être bradé au profit de l'industrie du médicament}

Un système de santé à deux vitesses semble maintenant implacablement établi. D'un côté, le système privé et les lobbies de l'argent qui permettent de gagner les avis des mieux informés; de l'autre, le système public qui contraint à accepter de consulter dans ses limites, c'est-à-dire en considérant que l'éthique du médecin le convainc de se

M.-F. Bacqué $(\bowtie)$

Rédactrice en chef de Psycho-Oncologie

Université de Strasbourg, département de psychologie,

12 rue Goethe, 67000 Strasbourg, France

e-mail : mfbacque@club-internet.fr former en permanence pour choisir les traitements les plus adaptés et renoncer à ceux qui n'ont pas fait leurs preuves. Or, les médecins sont aujourd'hui confrontés au même dilemme que les patients, lorsqu'à leur tour, ils découvrent qu'ils ne peuvent pas faire confiance aux experts et aux institutions expertales qui transmettent les indications de prescription. Ici, la question de l'économie est encore génératrice de fracture. Le médecin qui consacre du temps à sa formation ne peut pendant ce temps exercer (et donc gagner sa vie) et doit se fier aux lectures pour confirmer ou infirmer sa clinique. C'est ici que la revue Prescrire ${ }^{\circledR}$ intervient, ce journal qui signe chaque article du nom de la revue est engagé dans la politique de santé au point de risquer les attaques qui ne manquent pas, du côté des laboratoires, de se produire. Il ne s'agit pas ici de batailles d'experts, mais toujours d'enjeux économiques. Les laboratoires nous font pourtant croire qu'ils participent à une dynamique scientifique, alors qu'il s'agit bien de conserver des segments entiers du marché des consommateurs. Se pose alors une question de fond : quelle est la position des pouvoirs publics ? Ont-ils l'intention de continuer à soutenir l'industrie du médicament plutôt que la santé de la population française ? L'industrie des médicaments crée des emplois et génère des marges, mais ne pourrait-on choisir, à la manière des Américains aujourd'hui, de revenir à la source des problèmes de santé et de veiller aux conditions minimales d'alimentation pour prévenir les conséquences de la malnutrition ou plutôt de la dysnutrition de nos contemporains ? Si l'on finançait mieux l'agriculture « bio », si l'on organisait le sport au quotidien dans les villes, si l'on proposait des séances d'éducation et de réflexion dans les écoles, ne parviendrait-on pas à un état de santé qualitativement supérieur?

\section{Une prévention moins transparente}

La boutade qui consiste à dire que si l'on remboursait les fruits et les légumes, les chaussures de sport ou l'éducation diététique plutôt que les anorexigènes et les médicaments 
luttant contre la conséquence de notre alimentation pléthorique, la population se porterait mieux, est en passe de devenir une réalité aux États-Unis. Reprise par le marketing d'un géant de la distribution américaine, la devise " Aucune famille ne devrait avoir à choisir entre nourriture saine et nourriture bon marché » (Le Monde du 22 janvier 2011) [1] a été saluée par le gouvernement Obama. Le New York Times du 20 janvier 2011 commente la campagne de ce groupe de supermarchés de réduire le sel et le sucre des " milliers d'aliments ", en la comparant à une décision de la Food and Drug Administration...

Cette évolution pourrait de même porter sur deux thèmes de la politique publique en cancérologie. Dépister et guérir les cancers oui, certes, mais les prévenir ? N'est-ce pas au fond plus approprié pour tous? Dans le domaine de l'industrie agroalimentaire, la France est le premier pays d'Europe pour l'autorisation des produits chimiques sous forme de pesticides et d'engrais, alors que les études paraissent confirmer les dangers de mutation d'oncogènes et de développement de maladies neurodégénératives. La prévention semble hélas moins « vendable » en termes de communication politique que les victoires en termes de survie des patients malades. La prévention reste toujours hypothétique, tandis que la guérison ou la rémission peuvent se chiffrer en résultats objectifs (nombre de malades clairement obtenu). Nous ressentons le même sentiment d'abandon par les pouvoirs publics, lorsque la politique antitabac se voit totalement amoindrie avec des augmentations de tarifs ridicules (6\% de hausse n'ont aucune incidence sur la consommation), permettant à l'État comme aux cigarettiers de tirer de nouveaux profits, tandis que les jeunes fumeurs et surtout les femmes augmentent leur consommation de tabac pour la première fois depuis la loi Évin.

\section{Un laisser-mourir accompagné ou un faire mourir dépénalisé ?}

Les liens entre industriels et pouvoirs publics ne sont plus à démontrer. Le libéralisme est en effet bien établi hélas dans la vision à court terme de notre gouvernement. Augmenter le nombre d'accédants à la retraite ne peut plus, par exemple, être un objectif quand les soins en oncologie (principalement destinés aux plus âgés) sont si onéreux. Ce sentiment de plus en plus actuel soulève d'ailleurs d'autres craintes. On sait que le 25 janvier 2011, les propositions de loi n ${ }^{\circ} 659$ relative à l'aide active à mourir présentée par Jean-Pierre Godefroy, $\mathrm{n}^{\mathrm{o}} 65$ relative à l'aide active à mourir dans le respect des consciences et des volontés et $n^{\circ} 31$ relative à l'euthanasie volontaire présentées par Guy Fischer ont été refusées par le Sénat. On s'attend cependant à ce que leurs promoteurs reviennent à la charge. Dans le même registre, la légifération sur la fin de vie donnera un cadre qui n'a pas lieu d'être, puisque la loi $\mathrm{n}^{\mathrm{o}} 2005-370$ du 22 avril 2005, dite loi Léonetti, est suffisamment explicite sur l'abstention thérapeutique et la possibilité de "laisser mourir » dans des conditions très particulières de dialogue avec le malade, sa famille et ses soignants. Ici aussi une impression de collusion semble émerger.

La santé doit-elle être bradée aux industriels ? La vie ne peut-elle être qu'active et productive et sa fin futile ? L'accompagnement des plus âgés, des plus handicapés ou des plus malades n'a-t-il plus droit de cité, au prétexte qu'il n'est pas rentable? Le matérialisme cynique des lobbies de l'argent ne peut pas guider les politiques de santé, car il génère un doute qui débouche sur l'affrontement ou l'abandon.

Cet ensemble de faits, mis bout à bout, donne le vertige. Une vie vertigineuse, vécue à toute vitesse, bardée de technologies toujours plus coûteuses et rapidement obsolètes, aboutissant à une mort précipitée et une mémoire abandonnée... Il est temps de reprendre le contrôle pensent les populations de plus en plus informées. Les industriels peuvent dorénavant s'interroger sur le poids de la vertu retrouvée versus la perte de confiance, les réactions de panique ou la judiciarisation d'un monde livré à la bataille des effets indésirables. Le monde de la santé doit désormais compter avec l'empowerment du citoyen, dont la traduction signifie autant être rendu plus fort qu'informé. Le titre du livre de Stéphane Hessel avec lequel nous avons commencé l'année (Indignezvous, dans la collection au titre significatif Ceux qui marchent contre le vent) s'applique décidément à bien des champs de notre société [2]...

\section{Références}

1. Gatinois C, Lacombe C (2011) Walmart mise sur la nourriture saine pour soigner son image et contrer ses rivaux. Le Monde du samedi 22 janvier, p 16

2. Hessel S (2010) Indignez-vous. Eds. Indigène 